This item was submitted to Loughborough's Institutional Repository (https://dspace.lboro.ac.uk/) by the author and is made available under the following Creative Commons Licence conditions.

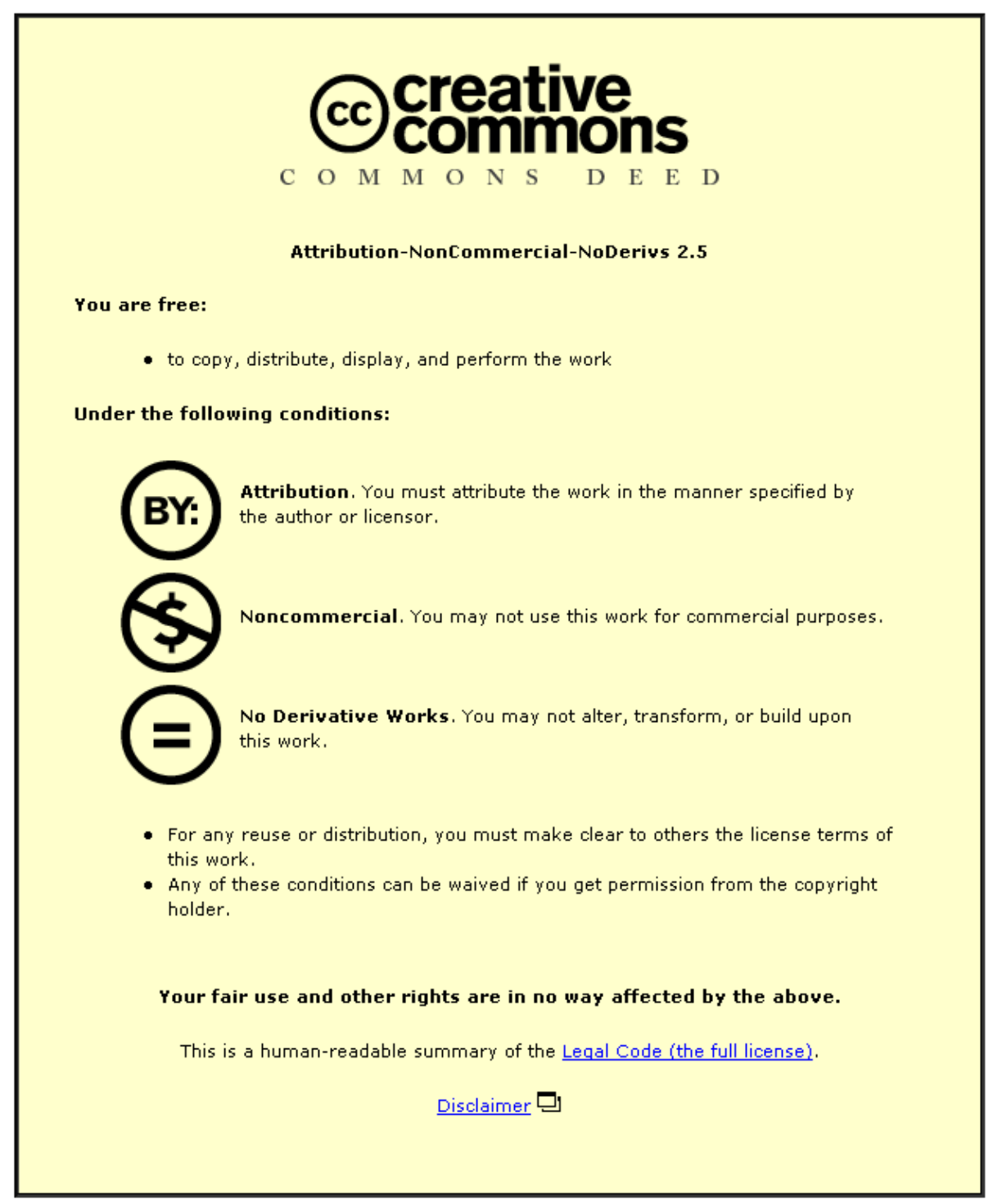

For the full text of this licence, please go to: http://creativecommons.org/licenses/by-nc-nd/2.5/ 


\title{
Multiple Reference Consistency Check for LAAS: a Novel Position Domain Approach
}

\author{
Liang $\mathrm{Li}^{1,2}$, Mohammed Quddus ${ }^{1}$, Stephen Ison $^{1}$ and Lin Zhao ${ }^{2}$ \\ ${ }^{1}$ Transport Studies Group \\ Department of Civil and Building Engineering \\ Loughborough University \\ Leicestershire LE11 3TU \\ United Kingdom \\ Phone: +44 (0)1509 228545 \\ Email:L.Li2@lboro.ac.uk \\ ${ }^{2}$ College of Automation \\ Harbin Engineering University \\ P.R. China \\ Phone: 0086-0451-82588866 \\ Email: zhaolin@hrbeu.edu.cn
}

\begin{abstract}
Since the traditional Maximum Likelihood (ML) based range domain Multiple Reference Consistency Check (MRCC) has limitations in satisfying the integrity requirement of CAT II/III for civil aviation, a Kalman filter based position domain method has been developed for fault detection and exclusion in Local Area Augmentation System (LAAS) MRCC process. The position domain approach developed in this paper seek to address the limitations of range domain based MRCC by focusing not only on improving the performance of the fault detection but also on the integrity risk requirement for MRCC. In addition, the issue of the stability of the Kalman filter in relation to the position domain approach is considered. GPS ranging corrections from multiple reference receivers are fused by the adaptive Kalman filter at the master station for detecting and excluding the single reference receiver' failure. The performance of the developed Kalman filter based MRCC algorithm has been compared with the traditional ML based method using experimental data. The results reveal that the Vertical Protection Level (VPL) is slightly better in the ML based method compared to the developed Kalman filter based approach under the faultfree case. However, the availability is better in the proposed method relative to the ML based approach under the single-fault case. In addition, a better fault-tolerant positioning result is obtained even if different fault types are considered under the single-fault case. In particular, the algorithm can be a candidate option as an augmentable complement for the traditional MRCC and can be implemented in a master station element of the LAAS integrity monitoring architecture.
\end{abstract}

Keywords: LAAS; MRCC; fault detection; integrity monitoring; GNSS 


\section{Introduction}

Global Navigation Satellite System (GNSS) has been employed as a pioneer ubiquitous positioning technology for navigation of moving objects (Misra and Enge 2001). One of its applications is in the area of civil aviation in which GNSS provides navigational aids in different flight phases. As a safety-of-life (SoL) critical application, the accuracy, integrity, continuity and availability (also known as Required Navigation Performance, RNP) requirements for navigation in civil aviation is more stringent relative to other non-safety critical applications. Due to the inherent limitations, a standalone GNSS (such as GPS) cannot satisfy the required navigational performance with sufficient accuracy and reliability, especially during the precision approach and landing phases of a flight. As such, the integrity requirement, which is directly related to safety, cannot be satisfied. One of the solutions to overcome this problem has been the LAAS (Enge 1999; Murphy and Imrich 2008; Walter et al. 2008). LAAS has been developed by the Federal Aviation Administration (FAA) in order to provide a high integrity positioning service for precision approach and landing operations of civil aviation. Each LAAS installation provides differential positioning services through a LAAS ground facility (LGF) which is located at the vicinity of the airport it serves and also includes multiple reference receivers, a master station facility and a very high frequency (VHF) data broadcast network. By monitoring the GPS signalin-space (SIS), LGF is able to detect and exclude faulty satellites while broadcasting high integrity range corrections within its service coverage (approximately a 20-30 mile). Civil aviation can use the broadcasted integrity information and pseudorange corrections to suppress the common errors between the LGF and a user. In the most stringent case (i.e. CAT II/III), it is necessary that the LAAS integrity monitoring architecture defined by the International Civil Aviation Organization (ICAO) should meet the following SIS requirements (ICAO SARPs 2007):

1. Integrity risk of $10^{-9}$ per approach with respect to a vertical alert limit (VAL) of approximately 5.3m and a time-to-alarm (TTA) of 1 to 2 sec.

2. Continuity risk of $4 \times 10^{-6}$ for any 15 sec of the approach.

Although the current integrity monitoring algorithms are striving to satisfy the RNP parameters at the CAT II/III precision approach, there are still a lack of widely acceptable and applicable methods. One of the main reasons for this is that there are some potential sources of integrity threats that need to be re-considered, especially the faults in the reference receivers (Khanafseh and Pervan 2011). To detect and mitigate the faults associated with the reference receivers in LAAS, the minimum aviation system performance standards (MASPS) for LAAS, which is documented in the radio technical commission of aeronautics (RTCA-DO245A), recommend that the master station should employ the so-called B-value to detect and exclude the outliers associated with the range domain corrections from the multiple reference receivers (e.g. Liu 1998; Wen et al. 2004). This kind of monitoring process is known as the traditional MRCC and its primary focus is the detection and exclusion of the faults arising from the multiple reference receivers. The B-value is computed for the hypothesis that a given reference receiver is 
faulty and fails to meet the required navigational performance. Hence the B-value expresses an estimation of the difference between the reference receivers for which it applies to and the consensus of the other reference receivers. The reference receiver in question is marked for the exclusion under the single-fault hypothesis if this difference exceeds a threshold (Gleason and Gebre-Egziabher 2009). The range domain based B-value fault detection scheme has however many disadvantages. For instance, the faults originating from either satellites or reference receivers could be responsible for an outlier in the B-value and be contributed to hazardous position error or misleading information. In addition to the faults in the satellites or reference receivers, there are other potential sources of faults (e.g. satellite clock-bias drift, satellite ephemeris type A and type B errors) (Tang et al. 2010) that may result in a conservative probability from which the detection threshold is normally being derived. Moreover, such faults could actually undermine the performance of traditional range domain MRCC. Therefore, if all kinds of fault are considered together, then it would be difficult to accurately express the correlation among different sources of fault. This may cause the MRCC performance to be inadequate to satisfy the RNP of CAT II/III. As far as the fault detection threshold is concerned, one of the determining factors in fault detection is the overlap between the probability density curves under the fault-free hypothesis and different fault hypotheses (Gustafsson 2005). This is the correlation of the test statistics under different hypotheses. It is always challenging to express the cross-correlations in terms of noise variance of raw measurements (Khanafseh and Pervan 2011).

The difficulty of formulating the correlation factor can also be a threat to LASS in terms of computation since the measurements from all reference receivers are coupled to each other in the range domain when processed in the master station (Khanafseh and Pervan 2011). This kind of threat can be simplified with the use of carrier phase based navigation because the noises contained in carrier phase measurements are much less. This will however be a problem for pseudorange based navigation as noises in pseudorange measurements are relatively large. Furthermore, Shively (1999) detailed and evaluated a series of range domain based MRCC methods with the conclusion that the performance of range domain based MRCC can be enhanced in terms of the trade-off between the integrity risk and continuity risk. Even the optimum B-value is derived from the minimum mean square error estimation criterion, the performance of such an optimum B-value based MRCC is almost the same as the traditional one (Kelly 2000).

In order to eliminate some of the disadvantages associated with the range domain based MRCC, the position domain based MRCC can be utilized and it has been proved that a better availability level can also be achieved by implementing the integrity monitoring for LAAS in the position domain with the multiple hypotheses solution separation algorithm (Pervan et al. 1998). However, there would appear to be a dearth of research relating to the application of the position domain method for fault detection and exclusion for MRCC. Therefore, this paper develops a position domain based method aimed at improving the MRCC performance for LAAS. A Kalman filter with the fading factor is applied to obtain an optimal test statistic for better MRCC performance and to adjust the filtering convergence procedure. The fading factor is optimal and adaptive for minimizing the integrity risk. 
The rest of paper is organized as follows. First, in section 2, ML based position domain MRCC is briefly introduced. Then the adaptive Kalman filter based MRCC is proposed and analyzed with the consideration of the stability of the Kalman filter and integrity risk. Finally, the results and the conclusions achieved from our experiment are discussed in sections 3 and 4 .

\section{Methods for the Position Domain Based MRCC}

A range of different estimation methods are available for MRCC. This includes ML (Liu 1998), Bvalue Bayesian exclusion (Shively 1999) and minimum mean square error (Kelly 2000). Each of the methods has their own advantages and disadvantages. Among these methods, one of the widely used methods is the ML. Due to the inherent limitations of the ML method (as explained later in this section), this paper introduces a novel position domain based MRCC for LAAS using an adaptive Kalman filter. This is fully discussed in this section. For completeness, the ML method for the position domain based MRCC is briefly discussed at first.

\subsection{The ML based position domain MRCC}

The traditional B-value can be implemented in both the range domain and the position domain. The weaknesses of traditional range domain based B-value are detailed in Wang et al. (2009). The better performance of the position domain method is illustrated in Pervan et al. (1998). Therefore, the position domain based B-value is analyzed to express the significance of the estimation method for the performance of MRCC.

Since the vertical performance is the primary concern in the precision approach of civil aviation, our work considers three pseudorange corrections associated with three sets of reference receivers that, when processed separately at the master station, generate three vertical position solution estimates defined as $y_{k}^{1}, y_{k}^{2}$, and $y_{k}^{3} . k$ is the index for $k$ th epoch. If these individual vertical position estimates are interpreted as individual "measurements" of position, their relationship to the true vertical position solution $\boldsymbol{x}_{\mathrm{v}, \mathrm{k}}$ can be given by:

$$
\underbrace{\left[\begin{array}{c}
y_{k}^{1} \\
y_{k}^{2} \\
y_{k}^{3}
\end{array}\right]}_{\boldsymbol{y}_{\mathrm{v}_{0}, k}}=\underbrace{\left[\begin{array}{c}
1 \\
1
\end{array}\right]}_{\boldsymbol{H}_{\mathrm{v}_{0}, k}} \boldsymbol{x}_{\mathrm{v}, k}+\underbrace{\left[\begin{array}{c}
\boldsymbol{v}_{r_{1}} \\
\boldsymbol{v}_{r_{2}} \\
\boldsymbol{v}_{r_{3}}
\end{array}\right]}_{\boldsymbol{v}_{\mathrm{v}_{0}, k}}
$$

where $\boldsymbol{y}_{\mathrm{v}_{0}}$ is the $3 \times 1$ vector parameters. $\quad \boldsymbol{H}_{\mathrm{v}_{0}}$ is the observation matrices. $\boldsymbol{v}_{\mathrm{v}_{0}}$ is the estimation error comprised of two random errors which come from master station and reference receivers. Subscript $\mathrm{v}_{0}$ and $\mathrm{v}_{i}(i=1,2,3)$ represent the fault-free and single-fault hypotheses respectively.

Since double reference receivers' faults fail to simultaneously generate no redundancy for integrity monitoring of the proposed system model, the single-fault case is therefore considered in this study. Under the single-fault case: 


$$
\left\{\begin{array}{l}
\boldsymbol{y}_{\mathrm{v}_{i}, k}=\boldsymbol{E}_{i} \boldsymbol{y}_{\mathrm{v}_{0}, k} \\
\boldsymbol{H}_{\mathrm{v}_{\mathrm{i}}, k}=\boldsymbol{E}_{i} \boldsymbol{H}_{\mathrm{v}_{0}, k}
\end{array}\right.
$$

where $\boldsymbol{E}=\boldsymbol{I}-\boldsymbol{e}_{i} \boldsymbol{e}_{i}^{\mathrm{T}} . \quad \boldsymbol{I}$ is the identity matrix, and $\boldsymbol{e}_{i}=\left[\begin{array}{lllllll}0 & \cdots & 0 & 1 & 0 & \cdots & 0\end{array}\right]^{\mathrm{T}} \cdot \boldsymbol{E}_{0}=\boldsymbol{I}$ is satisfied under the fault-free hypothesis.

The vertical estimation errors under different hypotheses are defined as:

$$
\left\{\begin{array}{l}
\tilde{\boldsymbol{x}}_{\mathrm{v}_{0}, k}^{\mathrm{L}}=\hat{\boldsymbol{x}}_{\mathrm{v}_{0}, k}^{\mathrm{L}}-\boldsymbol{x}_{\mathrm{v}, k} \\
\tilde{\boldsymbol{x}}_{\mathrm{v}_{i}, k}^{\mathrm{L}}=\hat{\boldsymbol{x}}_{\mathrm{v}_{i}, k}^{\mathrm{L}}-\boldsymbol{x}_{\mathrm{v}, k}
\end{array}\right.
$$

where $\hat{\boldsymbol{x}}_{\mathrm{v}_{0}, k}^{\mathrm{L}}$ and $\hat{\boldsymbol{x}}_{\mathrm{v}_{\mathrm{i}}, k}^{\mathrm{L}}$ are vertical position solutions from the Least Square (LS) estimation. $\tilde{\boldsymbol{x}}_{\mathrm{v}_{0}, k}^{\mathrm{L}}$ and $\tilde{\boldsymbol{x}}_{\mathrm{v}_{i}, k}^{\mathrm{L}}$ represent the vertical estimation error under the fault-free and fault case respectively. According to the definition of B-value (Liu 1998), the position domain based B-value can be expressed as:

$$
B_{\mathrm{v}_{i}, k}=\frac{1}{3} \sum_{j=1}^{3} \tilde{\boldsymbol{x}}_{\mathrm{v}_{j}, k}^{\mathrm{L}}-\frac{1}{2} \sum_{\substack{j=1 \\ j \neq i}}^{3} \tilde{\boldsymbol{x}}_{\mathrm{v}_{j}, k}^{\mathrm{L}}
$$

As seen from (4), the B-value is a ML estimation of positioning error from the applied reference receiver. One of the advantages of the ML based method is that it can be implemented with high computational effectiveness since a ML based method is actually a snapshot method. It has been proved that the ML based estimation accuracy is limited to the finite sample size used in the estimation process (Soong 2004), which means that more reference receivers were required to improve the ML based estimation accuracy. The data broadcasts rate has to be increased accordingly, and this will become a datalink bandwidth burden for the master station. The finite sample size demands a counterbalance between uncertainties in test statistics and susceptible of correlation coefficient between multiple reference receivers (Pervan and Sayim 2001). There are many candidate estimation methods that could deal with the sample size problem. One of the widely used methods is the Kalman filter that provides a better performance in the position domain (Khanafseh and Pervan 2011). In order to further investigate the weakness of the ML based values, the variance of and the covariance of B-value are given by:

$$
\left\{\begin{array}{l}
\operatorname{var}\left(B_{\mathrm{v}_{i}, k}\right)=\mathrm{E}\left[B_{\mathrm{v}_{i}, k} B_{\mathrm{v}_{i}, k}\right]=\frac{1}{6} \sigma_{i, k}^{2} \\
\operatorname{cov}\left(B_{\mathrm{v}_{i}, k}, B_{\mathrm{v}_{j}, k}^{i \neq j}\right)=\mathrm{E}\left[B_{\mathrm{v}_{i}, k} B_{\mathrm{v}_{j}, k}^{i \neq j}\right]=-\frac{1}{12} \sigma_{i, k}^{2}
\end{array}\right.
$$

where $\sigma_{i}$ represents the standard deviation of vertical position error with the application of pseudorange corrections. From .(5), $\operatorname{cov}\left(B_{\mathrm{v}_{i}, k}, B_{\mathrm{v}_{j}, k}^{i \neq j}\right)$ indicates that correlation exists between the B-values from different reference receivers. It can therefore be concluded that the outlier of 
one B-value would impose an impaction on the other B-values in the fault detection process. Equation (5) also implies that the position domain transformation is necessary but not sufficient for improving the performance of the traditional MRCC. We assume that the probability of false alarm for each reference receiver is the same, so the detection threshold can be calculated as follows:

$$
\frac{1}{3} \mathrm{P}_{\mathrm{fa}}=2 \mathrm{P}\left(\mathrm{v}_{0}\right) \cdot \int_{0}^{T_{\mathrm{M}}} \int_{T_{B}-x}^{T_{\mathrm{M}}}\left\{\frac{2 \sqrt{3}}{\pi \sigma_{i, k}^{2}} \cdot \exp \left[-\frac{4}{\sigma_{i, k}^{2}} \cdot\left(x^{2}+y^{2}+x y\right)\right]\right\} \mathrm{d} y \mathrm{~d} x
$$

where $\mathrm{P}_{\mathrm{fa}}$ is the probability of false alarm. $\mathrm{P}(\bullet)$ is the probability of the correspond case. $T_{\mathrm{M}}$ is the detection threshold for the ML based B-value. Eq. (6) implies that the correlation coefficient is one of the key factors to the system performance. Unfortunately the correlation is hard to appropriately express in terms of ramp errors contained in pseudorange corrections because a ML based method is independent of non-information priors. Furthermore, the effect of correlation between the fault-free hypothesis and fault hypothesis is another problem to be solved in order to improve the performance of the ML based MRCC.

To address the limitations of the ML based B-value noted above, a novel approach based on the Kalman filter is developed to deal with the problems of finite sample size and the expression of the correlation between the test statistics under different hypotheses, with the aim of satisfying the high integrity requirement for civil aviation.

\subsection{An adaptive Kalman filter based MRCC}

Based on the above analysis and evaluation of the traditional ML based position domain Bvalue, on one hand, the correlation between test statistics under different hypotheses should accurately be accounted for in enhancing the fault detection performance and on the other hand, the estimation criterion such as Kalman filter can be used to release the limitation induced by the ML based estimation method.

\subsubsection{Analysis of the Kalman filter based test statistics}

The Kalman filter based test statistic for MRCC can be defined as:

$$
\begin{gathered}
\boldsymbol{\beta}_{\mathrm{v}_{i}, k}=\tilde{\boldsymbol{x}}_{\mathrm{v}_{0}, k}-\tilde{\boldsymbol{x}}_{\mathrm{v}_{i}, k} \\
\boldsymbol{\beta}_{\mathrm{v}_{0}, k} \equiv 0
\end{gathered}
$$

where $\tilde{\boldsymbol{x}}_{\mathrm{v}_{0}, k}$ and $\tilde{\boldsymbol{x}}_{\mathrm{v}_{i}, k}$ are obtained from Kalman filter processing. It is obvious that the proposed test statistics $\boldsymbol{\beta}_{\mathrm{v}_{i}}$ is the positioning deviation in the master station caused by the exclusion of the position error from the candidate reference receiver $i$. The variance for the test statistics $\boldsymbol{\beta}_{\mathrm{v}_{i}}$ is as follows: 


$$
\begin{aligned}
\boldsymbol{C}_{\mathrm{v}_{i}, k} & =\mathrm{E}\left(\boldsymbol{\beta}_{\mathrm{v}_{i}, k} \boldsymbol{\beta}_{\mathrm{v}_{i}, k}^{\mathrm{T}}\right)=\mathrm{E}\left[\left(\tilde{\boldsymbol{x}}_{\mathrm{v}_{0}, k}-\tilde{\boldsymbol{x}}_{\mathrm{v}_{i}, k}\right)\left(\tilde{\boldsymbol{x}}_{\mathrm{v}_{0}, k}-\tilde{\boldsymbol{x}}_{\mathrm{v}_{i}, k}\right)^{\mathrm{T}}\right] \\
& =\boldsymbol{P}_{\mathrm{v}_{0}, k}-\boldsymbol{P}_{\mathrm{v}_{0, i}, k}-\boldsymbol{P}_{\mathrm{v}_{i, 0}, k}+\boldsymbol{P}_{\mathrm{v}_{i}, k}
\end{aligned}
$$

in which

$$
\begin{gathered}
\left\{\begin{array}{l}
\boldsymbol{P}_{\mathrm{v}_{0}, k}=\mathrm{E}\left(\tilde{\boldsymbol{x}}_{\mathrm{v}_{0}, k} \tilde{\boldsymbol{x}}_{\mathrm{v}_{0}, k}^{\mathrm{T}}\right) \\
\boldsymbol{P}_{\mathrm{v}_{i}, k}=\mathrm{E}\left(\tilde{\boldsymbol{x}}_{\mathrm{v}_{i}, k} \tilde{\boldsymbol{x}}_{\mathrm{v}_{i}, k}^{\mathrm{T}}\right)
\end{array}\right. \\
\boldsymbol{P}_{\mathrm{v}_{0, i}, k}=\mathrm{E}\left(\tilde{\boldsymbol{x}}_{\mathrm{v}_{0}, k} \tilde{\boldsymbol{x}}_{\mathrm{v}_{\mathrm{i}}, k}^{\mathrm{T}}\right)=\boldsymbol{P}_{\mathrm{v}_{i, 0}, k}^{\mathrm{T}}
\end{gathered}
$$

It is noted that Eq. (8) yields $C_{\mathrm{v}_{0, i}, \mathrm{k}} \equiv 0$, which means that the limitation of correlation between the fault-free hypothesis and fault hypothesis is liberated by the proposed test statistics. It has been proved that the cross-correlation $\boldsymbol{P}_{\mathrm{v}_{0, i}, k}$ is quite challenging to obtain (Khanafseh and Pervan 2011). In the following analysis, it will be proved that the cross-correlation can be accurately expressed with the application of the new proposed $\boldsymbol{\beta}_{\mathrm{v}_{i}}$.

The initial condition and covariance for position solution estimation satisfy:

$$
\begin{gathered}
\hat{\boldsymbol{x}}_{\mathrm{v}_{0}, 0}=\hat{\boldsymbol{x}}_{\mathrm{v}_{i}, 0}=\mathrm{E}\left\{\boldsymbol{x}_{\mathrm{v}, 0}\right\}=\overline{\boldsymbol{x}}_{0} \\
\boldsymbol{P}_{\mathrm{v}_{0}, 0}=\boldsymbol{P}_{\mathrm{v}_{i}, 0}=\boldsymbol{P}_{\mathrm{v}_{0, i}, 0}=\overline{\boldsymbol{P}}_{0}
\end{gathered}
$$

The estimation error under different hypotheses in the Kalman filtering process is given by:

$$
\tilde{\boldsymbol{x}}_{\mathrm{v}_{i}, k}=\boldsymbol{x}_{\mathrm{v}, k}-\hat{\boldsymbol{x}}_{\mathrm{v}_{i}, k}=\left(\boldsymbol{I}-\boldsymbol{K}_{\mathrm{v}_{i}, k} \boldsymbol{H}_{\mathrm{v}_{i}, k}\right) \tilde{\boldsymbol{x}}_{\mathrm{v}_{i}, k \mid k-1}-\boldsymbol{K}_{\mathrm{v}_{i}, k} \boldsymbol{v}_{\mathrm{v}_{i}, k}
$$

The following can be obtained by substituting Eq. (14) into Eq. (11)

$$
\boldsymbol{P}_{\mathrm{v}_{0, i}, k}=\mathrm{E}\left(\tilde{\boldsymbol{x}}_{\mathrm{v}_{0}, k} \tilde{\boldsymbol{X}}_{\mathrm{v}_{i}, k}^{\mathrm{T}}\right)=\boldsymbol{\Psi}_{\mathrm{v}_{0}, k \mid k-1} \boldsymbol{P}_{\mathrm{v}_{0, i}, k-1} \boldsymbol{\Psi}_{\mathrm{v}_{i}, k \mid k-1}^{\mathrm{T}}+\boldsymbol{W}_{\mathrm{v}_{0}, k} \boldsymbol{Q}_{k} \boldsymbol{W}_{\mathrm{v}_{i}, k}^{\mathrm{T}}+\boldsymbol{K}_{\mathrm{v}_{0}, k} \boldsymbol{R}_{\mathrm{v}_{0, i}, k} \boldsymbol{K}_{\mathrm{v}_{i}, k}^{\mathrm{T}}
$$

where

$$
\left\{\begin{array}{l}
\boldsymbol{W}_{\mathrm{v}_{i}, k}=\boldsymbol{I}-\boldsymbol{K}_{\mathrm{v}_{i}, k} \boldsymbol{H}_{\mathrm{v}_{i}, k} \\
\boldsymbol{\Psi}_{\mathrm{v}_{i}, k \mid k-1}=\boldsymbol{W}_{\mathrm{v}_{i}, k} \boldsymbol{\Phi}_{k \mid k-1} \\
\boldsymbol{R}_{\mathrm{v}_{0, i}, k}=\mathrm{E}\left(\boldsymbol{v}_{\mathrm{v}_{0}, k} \boldsymbol{v}_{\mathrm{v}_{i}, k}^{\mathrm{T}}\right)=\boldsymbol{R}_{\mathrm{v}_{0}, k} \boldsymbol{E}_{i}
\end{array}\right.
$$

The dynamic matrices $\boldsymbol{\Phi}_{k \mid k-1}$ and $\boldsymbol{K}_{\mathrm{v}_{i}, k}$ is known and already evaluated from master station's Kalman filter update.

According to the Kalman filter, the following can be obtained

$$
\boldsymbol{P}_{\mathrm{v}_{0}, k}=\boldsymbol{W}_{\mathrm{v}_{0}, k}\left(\boldsymbol{\Phi}_{\mathrm{v}_{0}, k \mid k-1} \boldsymbol{P}_{\mathrm{v}_{0}, k-1} \boldsymbol{\Phi}_{\mathrm{v}_{0}, k \mid k-1}^{\mathrm{T}}+\boldsymbol{Q}_{k}\right)
$$


The mathematical induction method used to prove $\boldsymbol{P}_{\mathrm{v}_{0, i}, k}=\boldsymbol{P}_{\mathrm{v}_{0}, k}$ is presented in appendix A. By substituting $\boldsymbol{P}_{\mathrm{v}_{0, i}, l}=\boldsymbol{P}_{\mathrm{v}_{0}, l}$ into Eq. (9), the following equation emerges:

$$
\boldsymbol{C}_{\mathrm{v}_{i}, k}=\boldsymbol{P}_{\mathrm{v}_{i}, k}-\boldsymbol{P}_{\mathrm{v}_{0}, k}^{\mathrm{T}}
$$

Since $\boldsymbol{P}_{\mathrm{v}_{0}, k}$ and $\boldsymbol{P}_{\mathrm{v}_{i}, k}$ can be available from the Kalman filtering processing, $\boldsymbol{C}_{\mathrm{v}_{i}, k}$ can be easily calculated as the difference of the two variance matrices. This simplification yields considerable computation savings. Another particular significance is that the cross-correlation can be accurately expressed by the position domain based test statistics.

The fault detection and exclusion process should be continued to complete the MRCC after choosing the test statics for the MRCC.

\subsubsection{Fault detection and exclusion}

After the analysis of the Kalman filter based test statistics, the fault detection and exclusion threshold would be another important factor for the performance of fault detection. Assuming that $\boldsymbol{\beta}_{\mathrm{v}_{i}}$ follows a Gaussian distribution (The subscript $k$ is not used in the following equations for clarity):

$$
f_{z}\left(\boldsymbol{\beta}_{\mathrm{v}_{i}} \mid \mathrm{v}_{i}\right)=\frac{1}{\sqrt{2 \pi \cdot \boldsymbol{C}_{\mathrm{v}_{i}}}} \exp \left(-\frac{1}{2} \mathbf{z}^{\mathrm{T}} \boldsymbol{C}_{\mathrm{v}_{i}}^{-1} \mathbf{z}\right)
$$

As stated, the GPS based positioning errors do not always follow the Gaussian distribution perfectly (Misra and Enge 2001), the Gaussian distribution model may not be accurate enough to characterize the test statistics. However, the sigma inflation can be applied to overbound the Gaussian assumption. A reasonable sigma inflation factor $\gamma_{i}$ is necessary to overbound the nonGaussian and non-zero mean deviation of $\boldsymbol{\beta}_{\mathrm{v}_{i}}$ and satisfy the integrity risk requirement.

It is noted that under the single-fault hypothesis, the fault detection threshold can also be the exclusion threshold because the individual residual method is used in this study. For convenient comparison, $\left|\boldsymbol{\beta}_{\mathrm{v}_{i}}\right|$ is chosen to be compared with the detection and exclusion threshold $T_{\mathrm{K}, i}$. So the detection threshold under the single-fault hypothesis can be achieved by the following equation:

$$
\begin{aligned}
\mathrm{P}_{\mathrm{fa}}= & \sum_{i=1}^{3} \mathrm{P}\left(\mathrm{v}_{0}\right)\left[\mathrm{P}\left(\left|\boldsymbol{\beta}_{\mathrm{v}_{i}}\right|>T_{\mathrm{K}, i}\right)\right]= \\
& \sum_{i=1}^{3} \mathrm{P}\left(\mathrm{v}_{0}\right)\left[\int_{T_{\mathrm{K}, i}}^{\infty} f_{z}\left(\boldsymbol{\beta}_{\mathrm{v}_{i}} \mid \mathrm{v}_{i}\right) \mathrm{d} z+\int_{-\infty}^{-T_{\mathrm{K}, i}} f_{z}\left(\boldsymbol{\beta}_{\mathrm{v}_{i}} \mid \mathrm{v}_{i}\right) \mathrm{d} z\right]
\end{aligned}
$$

By comparing the resulting threshold $T_{\mathrm{K}, i}$ and the predefined test statistic $\left|\boldsymbol{\beta}_{\mathrm{v}_{i}}\right|$, the detection and exclusion process can be implemented. 


\subsection{Limitations and Improvements for the Kalman filter based MRCC}

Although the proposed Kalman filter based test statistic can deal with the problem of correlation between the test statistics as well as the limitation of finite sample size, there are many underlying factors to be addressed to make the algorithm more suitable for implementation.

\subsubsection{The stability of the Kalman filter}

It is difficult to precisely characterize the correlation between test statistics under different hypotheses in the traditional MRCC, but the correlation can be correctly expressed in the proposed test statistic as seen from Eq. (17). However, the proposed fault detection may also induce the high probability of missed detection or false alarm. The reasons are divided into two types:

(1) If the filter output is converged, it means that $\left\|\boldsymbol{P}_{\mathrm{v}_{i}}\right\| \leq \xi$ is satisfied for some $\xi>0$. Then $\left\|\boldsymbol{P}_{\mathrm{v}_{0}}\right\| \leq \xi$ is also satisfied according to Eq. (17). Given the $\xi$ is small enough, the convergence of $\boldsymbol{P}_{\mathrm{v}_{i}}$ and $\boldsymbol{P}_{\mathrm{v}_{0}}$ lead filter output to rely on the prior measurements with much less weighting on the new input measurements, even if there is a fault contained in the measurements. This kind of convergence actually is pseudo-convergence and divergence of Kalman filter because the Kalman filter loses the ability of tracking new input measurements. This indicates that the pseudo-convergence of the Kalman filter will result in a high probability of missed detection.

(2) Similarly, if the system model is mismatch with the new input measurements and cause $\boldsymbol{P}_{\mathrm{v}_{i}}$ and $\boldsymbol{P}_{\mathrm{v}_{0}}$ are to diverge, the filtering divergence will lead to worse availability because the positioning estimation is misleading in this situation.

It can be concluded that the probability of missed detection and false alarm is caused by the stability of the Kalman filter in essence. In the filtering process, the state estimation is a combination of the prior state estimate and the new input measurements through the Kalman filtering gain. The prior state estimation will dominate the state estimation if $\left\|\boldsymbol{P}_{\mathrm{v}_{i}}\right\| \leq \xi$ and $\xi$ is small enough, whereas the input measurements are underweighted in the filtering process. Therefore, the weighted fading factor can be applied to suppress the effect of Kalman filter stability on the fault detection as stated above. The adaptive Kalman filtering gain can be calculated as:

$$
\overline{\boldsymbol{K}}_{\mathrm{v}_{i}, k}=\frac{1}{\alpha_{\mathrm{v}_{i}, k}^{2}} \boldsymbol{P}_{\mathrm{v}_{i}, k} \boldsymbol{H}_{\mathrm{v}_{i}, k}^{\mathrm{T}} \boldsymbol{R}_{\mathrm{v}_{i}, k}^{-1} \quad(i=0,1,2,3)
$$

where $\alpha_{\mathrm{v}_{i}}$ is the fading factor for weighting the input measurements. It is obvious that the fading factor will not influence the Kalman filter based test statistics analysis. $\left|\alpha_{\mathrm{v}_{i}}\right| \leq 1$ need to be satisfied with the purpose of tracking the new input measurements by overweighting the effect of current measurements and underweighting the effect of prior measurements in the Kalman filtering process. If the system model mismatch with the new input measurements, the adaptive Kalman filter will track the new input measurements by adjusting the fading factor. In this way, the 
developed adaptive Kalman filter achieves the better performance. It is noted that $\boldsymbol{P}_{\mathrm{v}_{0, i}}$ and $\boldsymbol{P}_{\mathrm{v}_{0}}$ can be weighted with the same factor $\alpha_{\mathrm{v}_{0}}$ as the benefit of the equality Eq. (17):

$$
\overline{\boldsymbol{C}}_{\mathrm{v}_{i}}=\frac{\gamma_{i}^{2}}{\alpha_{\mathrm{v}_{i}}^{2}} \boldsymbol{P}_{\mathrm{v}_{i}}-\frac{\gamma_{0}^{2}}{\alpha_{\mathrm{v}_{0}}^{2}} \boldsymbol{P}_{\mathrm{v}_{0}}^{\mathrm{T}}
$$

It is noted that the pre-specified $C_{\mathrm{v}_{i}}$ for the determination of the fault detection threshold should be replaced with the weighted variance matrices $\overline{\boldsymbol{C}}_{\mathrm{v}_{i}}$. An improper fading factor will induce $\overline{\boldsymbol{C}}_{\mathrm{v}_{i}}$ lose the positive definite and impose a detrimental impact on the availability and the stability of the Kalman filter based MRCC algorithm. There are many candidate algorithms for selecting adaptive factors, the integrity performance however is the primary concern of the proposed Kalman filter based MRCC algorithm. Next the selecting of $\alpha_{\mathrm{v}_{i}}$ will be analyzed with consideration of the integrity risk.

\subsubsection{Integrity risk}

Although the fading factor can deal with the filter stability problem, the introduction of such a factor will undoubtedly impose a threat to the availability of the proposed MRCC algorithm. The VPL can be expressed as follows:

$$
V P L_{k}=\max _{i}\left(\left|\beta_{\mathrm{v}_{i}, k}\right|+\kappa_{\mathrm{md}} \overline{\boldsymbol{C}}_{\mathrm{v}_{i}, k}\right)
$$

where $\kappa_{\mathrm{md}}$ is the standard deviation corresponding to the probability of missed detection $\mathrm{P}_{\mathrm{md}}$. It can be seen from Eq. (22) that the availability level is degraded because $\overline{\boldsymbol{C}}_{\mathrm{v}_{i}} \geq \boldsymbol{C}_{\mathrm{v}_{i}}$ is satisfied. To keep a balance between the ability of tracking new measurements and the integrity risk requirement of the LAAS, the pre-specified parameter $\alpha_{\mathrm{v}_{i}}$ in the adaptive Kalman filter will be an important buffer in obtaining the optimal MRCC performance.

According to the integrity risk concept (Braff 2001), the predefined integrity risk $I_{v}$ can be calculated as follows:

$$
\mathrm{I}_{\mathrm{v}}=\mathrm{P}\left(\left|\boldsymbol{x}_{\mathrm{v}}-\hat{\boldsymbol{x}}_{\mathrm{v}}\right|>\mathrm{VAL}\right)=\int_{\hat{\boldsymbol{x}}_{\mathrm{v}}+\mathrm{VAL}}^{\infty} f_{\boldsymbol{x}_{\mathrm{v}}}\left(\tilde{\boldsymbol{x}}_{\mathrm{v}}\right) \mathrm{d} \tilde{\boldsymbol{x}}_{\mathrm{v}}+\int_{-\infty}^{\hat{\boldsymbol{x}}_{\mathrm{v}}-\mathrm{VAL}} f_{\boldsymbol{x}_{\mathrm{v}}}\left(\tilde{\boldsymbol{x}}_{\mathrm{v}}\right) \mathrm{d} \tilde{\boldsymbol{x}}_{\mathrm{v}}
$$

where $\hat{\boldsymbol{x}}_{\mathrm{v}}$ is the arbitrary vertical position estimation with estimation error $\tilde{\boldsymbol{x}}_{\mathrm{v}}$, which follows the Gaussian distribution of $f_{x_{v}}\left(\tilde{\boldsymbol{x}}_{\mathrm{v}}\right)$. The integrity risk can be expressed under different hypotheses by using the Bayesian formula with consideration of the fading factor:

$$
\mathrm{I}_{\mathrm{v}}=\sum_{i=0}^{3} \mathrm{P}\left(\mathrm{v}_{i}\right)\left[\int_{\hat{\boldsymbol{x}}_{\mathrm{v}}+\mathrm{VAL}}^{\infty} \mathrm{N}_{\boldsymbol{x}}\left(\hat{\boldsymbol{x}}_{\mathrm{v}_{i}}, \frac{1}{\alpha_{\mathrm{v}_{i}}^{2}} \gamma_{i}^{2} \boldsymbol{P}_{\mathrm{v}_{i}}\right) \mathrm{d} \tilde{\boldsymbol{x}}_{\mathrm{v}}+\int_{-\infty}^{\hat{\boldsymbol{x}}_{\mathrm{v}}-\mathrm{VAL}} \mathrm{N}_{\boldsymbol{x}}\left(\hat{\boldsymbol{x}}_{\mathrm{v}_{i}}, \frac{1}{\alpha_{\mathrm{v}_{i}}^{2}} \gamma_{i}^{2} \boldsymbol{P}_{\mathrm{v}_{i}}\right) \mathrm{d} \tilde{\boldsymbol{x}}_{\mathrm{v}}\right]
$$


in which $\mathrm{P}\left(\mathrm{v}_{i}\right)$ is the predetermined probability of reference receiver $i$ failure. The integrity risk requirement can be met and minimized by differentiating Eq. (24) with respect to $\alpha_{\mathrm{v}_{i}}$. Setting the result equal to zero yields:

$$
\begin{aligned}
& \sum_{i=0}^{3} \mathrm{P}\left(\mathrm{v}_{i}\right)\left\{\frac{\mathrm{VAL}}{\alpha_{\mathrm{v}_{i}} \gamma_{i} \sqrt{2 \pi \boldsymbol{P}_{\mathrm{v}_{i}}}} \exp \left[\left(\frac{\alpha_{\mathrm{v}_{i}} \mathrm{VAL}+\alpha_{\mathrm{v}_{i}} \beta_{\mathrm{v}_{i}}}{\gamma_{i} \sqrt{2 \boldsymbol{P}_{\mathrm{v}_{i}}}}\right)^{2}+\exp \left(\frac{\alpha_{\mathrm{v}_{i}} \mathrm{VAL}-\alpha_{\mathrm{v}_{i}} \beta_{\mathrm{v}_{i}}}{\gamma_{i} \sqrt{2 \boldsymbol{P}_{\mathrm{v}_{i}}}}\right)^{2}\right]+\right. \\
&\left.\frac{1}{2 \alpha_{\mathrm{v}_{i}}^{2}}\left[\operatorname{erfc}\left(\frac{\alpha_{\mathrm{v}_{i}} \mathrm{VAL}+\alpha_{\mathrm{v}_{i}} \beta_{\mathrm{v}_{i}}}{\gamma_{i} \sqrt{2 \boldsymbol{P}_{\mathrm{v}_{i}}}}\right)+\operatorname{erfc}\left(\frac{\alpha_{\mathrm{v}_{i}} \mathrm{VAL}-\alpha_{\mathrm{v}_{i}} \beta_{\mathrm{v}_{i}}}{\gamma_{i} \sqrt{2 \boldsymbol{P}_{\mathrm{v}_{i}}}}\right)\right]\right\}=0
\end{aligned}
$$

(for more details, see Appendix B)

$\alpha_{\mathrm{v}_{i}} \in[-1,0)$ can be induced from Eq.(25). There are many kinds of searching algorithms for the above equation such as simulated annealing and artificial neural network. But these methods are time-consuming and even are a potential threat to the TTA requirement. In this paper, we use a numerical simulation method to search for the optimum fading factor.

\section{Experiment and Discussion}

A MRCC test bed was constructed on a building roof at Loughborough University so as to perform a real-time test and verify the proposed algorithm. In this experiment, four GPS receivers were used to simultaneously provide pseudoranges, carrier phase and position solution with $1 \mathrm{~Hz}$ sample frequency. One of the receivers was considered as the master station (located at 5245'42.468315", -1²14'29.607147", 128.5545m) and the other three receivers treated as the reference receivers. The location of each of the reference receivers was precisely determined by performing carrier phase differential positioning. The configuration of the test bed is shown in Fig. 1. The total duration of data collection was approximately 5,000s.

A simulation was performed to assess the proposed adaptive Kalman filter based MRCC (AKFM). To investigate the performance of the developed algorithm, the traditional ML based MRCC (MLM) was also implemented in parallel. The smoothing time constants in the master station and reference receivers were set up to 100s. A position-velocity model is used as the state equation for the master station (Crassdis and Junkins 2004). The probability of the single-fault hypothesis $\mathrm{P}\left(\mathrm{v}_{i}\right)$ was set to $10^{-5}$. $\kappa_{\mathrm{md}}$ was 6.327 (fault-free) or 4.125 (single-fault), given the integrity risk requirement was $10^{-9}$. The probability of fault-free alarm $\mathrm{P}_{\mathrm{fa}}$ was set to $10^{-7}$. The searching step length for the optimum fading factor was set up to 0.1 to release the computation burden. The performance of these two algorithms is compared with respect to the fault-free hypothesis and single-fault hypothesis. 


\subsection{Results and analysis: fault-free}

To obtain the sigma inflation factor for the non-perfect distribution of test statistics $\beta_{\mathrm{v}_{i}}$ under the Gaussian assumption, the sigma inflation of $\tilde{\boldsymbol{x}}_{\mathrm{v}_{i}}$ is applied because linear combination of $\quad \tilde{\boldsymbol{x}}_{\mathrm{v}_{i}}$ would guarantee a Gaussian distribution of $\beta_{\mathrm{v}_{i}}$. The Quantile-Quantile (QQ) plot of $\tilde{\boldsymbol{x}}_{\mathrm{v}_{i}}$ is presented in Fig. 2. This analysis is necessary since the Gaussian assumption was made for determining the detection and exclusion threshold. This result suggests that the distribution becomes more Gaussian-like as it gets closer to a straight line and a larger slope indicates a larger standard deviation. As seen in Fig. 2, the distribution of $\tilde{\boldsymbol{x}}_{\mathrm{v}_{i}}$ is a combination of Gaussianmixture model, which includes the non-Gaussian and non-zero mean value other than the Gaussian. This may be caused by the low sample size (i.e. pseudorange and carrier phase measurements to get the position solution). Through the analysis of the distribution, the sigma inflation factors are as follows:

$$
\left\{\begin{array}{l}
\gamma_{0}=1.25 \\
\gamma_{1}=1.35 \\
\gamma_{2}=1.20 \\
\gamma_{3}=1.21
\end{array}\right.
$$

The VPL performance of MLM and AKFM is investigated to show the effect of the adaptive fading factor under the fault-free case. The VPL comparison under the fault-free condition is shown in Fig. 3, in which Fig. 3a shows the VPL scenario in the real-time test process, and Fig. 3b shows the distribution of VPLs from the two algorithms. It can be seen that MLM based VPL is smaller than that of AKFM, which means the developed AKFM comes with the cost of aggravated VPL. However the VPL loss from the AKFM is still acceptable (VAL is set up to 5.3m according to ICAO SARPs (2007)). Therefore the AKFM can be considered a good option to improve the performance of traditional MRCC.

\subsection{Results and analysis: single-fault}

In evaluating the proposed MRCC algorithm, the manual faults were injected into the pseudorange corrections achieved from the reference receivers. Through the post-processing of the collected data, the standard deviation $\sigma$ of range domain based B-value is assumed as $2.5 \mathrm{~m}$. To ensure that the injected fault could cause the anomaly of pseudorange corrections, the peak amplitude of the input fault is set up to $2 \sigma$. Most of the existing MRCC studies focused on monitoring the step fault contained in the pseudorange corrections. However, some kind of error sources, such as satellite clock-bias drift, could induce ramp error in the pseudorange corrections, which is a threat to the traditional MRCC. In this study, different types of fault are generated to test the flexibility of the proposed algorithm such as the step fault, slow ramp fault and fast ramp fault. The details of generated fault are shown in Fig. 4.

In order to demonstrate the necessity for introducing an adaptive Kalman filter for MRCC, the positioning error from using corrections from different reference receivers are shown in Fig. 5. 
E4Rf1, E4Rf2 and E4Rf3 indicate the positioning error after pseudorange corrections corresponding to the three reference receivers. AKF and TKF represent the adaptive and traditional Kalman filters respectively. It can be seen that positioning error from the TKF cannot react accurately to the input faults, and actually is a kind of divergence. This may induce a higher probability of missed detection. The AKF could however track the anomaly of the input measurements because of the introduction of the adaptive fading factor.

The MLM and AKFM based test statistics and threshold from the three reference receivers in the fault detection process is shown in Fig. 6a and Fig. 6b respectively. There are multiple solutions for $T_{\mathrm{K}, i}$ in the simulation. Here the solution that satisfies $T_{\mathrm{K}, i}=T_{\mathrm{K}}(i=1,2,3)$ is selected as the unique solution.

As shown in Fig. 6, the MLM has a quicker response in detecting the step fault in the first few epochs, as the MLM is a snapshot method with no prior measurements. The AKFM, on the other hand, has a few seconds latency in detecting the input fault due to the weighting effect of prior measurements in the Kalman filter. This kind of latency should not be a problem because the fault detecting operation is carried out in 15s (per approach), which is long enough for the AKFM to response. However, the outlier of the MLM based test statistic will affect the others since the MLM based test statistics have a strong correlation. This kind of correlation is a threat to the individual residual based method if the correlation cannot be properly expressed. In comparison, the AKFM based test statistics presents a good performance for accurately addressing the correlation between different statistics. The AKFM can be anticipated to have a better fault detection process. It can therefore be concluded that the cross-correlation between the fault-free hypothesis and the fault hypothesis is more accurately expressed in the AKFM than that in MLM. To have further insight into the fault detection performance, the fault detection result is shown in Fig. 7 and Table 1.

As can be seen in Fig.7 and Table 1, four cases (i.e. A, B, C and D) are considered. The developed AKFM has nearly the same performance with that of the MLM in the step fault and no fault situations. But when it involves the slow ramp fault and fast ramp fault, the AKFM offers a better fault detection performance than that of MLM. It is noted that both AKFM and MLM fail to detect small-bias faults at the beginning of the detecting process under the case of slow ramp fault. Neither MLM nor AKFM could detect a fault until the amplitude of the fault increases to 1.5m.

The correct fault detection ratio between different fault types comparison are shown in Table 2 in order to make a numerical comparison and express the superior performance of AKFM,

From Table 2, it can be seen that the performance of correct fault detection from the AKFM and MLM is similar in the case of no fault. There are however marked differences in the performances of correct fault detections for all other cases. The correct fault detection rate is much higher (14\% to $31 \%$ ) in the AKFM relative to the MLM. It reveals that the AKFM could improve the performance of ML based MRCC, especially when dealing with ramp fault. It is because the cross-correlation can be expressed accurately by using AKFM that even the ramp fault may cause the cross-correlation to fluctuate.

To further assess the effectiveness of AKFM in positioning, Fig. 8 shows the vertical positioning error and VPL after the fault detection and exclusion. Given the superior fault 
detection and exclusion performance of the AKFM, the AKFM provides a smaller positioning error and a better VPL level. This is because the AKFM succeeds in excluding the higher number of faults therefore the positioning result can be fault-tolerant. Table 3 shows the CEP 95\% of the vertical positioning results. As can be seen in Table 3, the positioning accuracies of AKFM are found to be $77 \%$ (no fault), 32\% (step fault), 91\% (slow ramp fault) and 52\% (fast ramp fault) and these are smaller than those of MLM. This superior positioning performance benefits from the better fault detection performance of AKFM.

Since the study tries to satisfy the integrity monitoring requirement of CAT П/III, availability results from AKFM and MLM are shown in Table 4. It is clear from Table 4 that MLM fails to satisfy the integrity requirement of CAТ П/III, especially in the cases of ramp faults. Meanwhile, the availability enhances significantly in all cases when the AKFM is employed. This availability benefit is not only from the better fault detection performance, but also the appropriate account for the correlation efficient as shown in Eq. (17) and the adaptive fading factor for minimizing the integrity risk. The AKFM is however found not to be capable of satisfying the availability requirement of CAT П/III in the case of slow ramp fault. This is mainly due to the fact that AKFM is unable to detect faults related to small-bias.

\section{Conclusion}

An adaptive Kalman filter based position domain MRCC algorithm for fault detection and exclusion in LAAS has been developed, implemented and tested. The effect of the Kalman filter stability and integrity risk has been directly evaluated under the single-fault hypothesis. The developed adaptive Kalman filter based MRCC has been augmented with the optimal adaptive factor so as to minimize integrity risk.

The results from the real-world MRCC experiment have indicated that the VPL of the proposed method under the fault-free case is worse, but the VPL from the developed method is still acceptable under the SIS requirement of ICAO. Furthermore, because the developed adaptive Kalman filter based position domain MRCC has a better fault detection performance than the ML based position domain MRCC, the former one could provide a more fault-tolerant vertical positioning result and a better availability that could satisfy the requirement of CAT II/III with the exception of the slow ramp fault case. Although the adaptive Kalman filter based MRCC has latency in detecting the ramp faults, this latency is tolerable under the current LAAS establishment. It can be concluded that the proposed MRCC algorithm can be a candidate option to improve the traditional MRCC methodology.

In the future, sharper and more sensitive algorithms should be developed for dealing with detecting small-bias in the slow ramp fault, and offsetting the fault detecting delay. In addition, since our algorithm is based on single-fault detection and exclusion, it should be expanded to detect and exclude simultaneously multiple faults.

\section{Acknowledgements}

We gratefully acknowledge the financial support from the Chinese Scholarship Council (CSC) which allows Liang Li to carry out research for 1-year at Loughborough University, UK. 
We would like to thank Dr. Rene Wackrow, Dr. Yuheng Zheng and Mr. Nick Rodgers for their assistance in constructing the MRCC test bed and collecting the GPS data.

\section{Reference}

Braff R (2001) LAAS performance for terminal area navigation. In: Proceedings of the 57th Annual Meeting of The Institute of Navigation, NM, pp 252-262

Crassdis J, Junkins J (2004) Optimal estimation of dynamical systems. Capman \& Hall/CRC, New York Enge P (1999) Local area augmentation of GPS for the precision approach of aircraft. Proceedings of IEEE 87(1): 111-132

Gleason S, Gebre-Egziabher D (2009) GNSS application and methods. Artech House, New York Gustafsson F (2005) Adaptive filtering and change detection. John Wiley, New York International Civil Aviation Organization (ICAO) Aeronautical Telecommunications in Annex 10 to the Convention on International Civil Aviation International Standards and Recommended Practices (SARPs) (2007), Volume I (Radio Navigation Aids), Canada

Kelly R (2000) Comparison of LAAS B-values with linear model optimum B-values. The Journal of the Institute of Navigation 47(2):143-156

Khanafseh S, Pervan B (2011) Detection and mitigation of reference receiver faults in differential carrier phase navigation systems. Illinois Institute of Technology NavLabWeb.

http://mmae.iit.edu/ gps/publications/open/samer_journal\%20IEEE_2010.pdf. Accessed by 25 March 2010.

Liu F (1998) Analysis of integrity monitoring for the local area augmentation system using global navigation satellite system. Ph.D Dissertation, Ohio University

Misra P, Enge P (2001) Global positioning system: signals, measurements, and performance. Ganga-Jamuna Press, Lincoln, Mass.

Murphy T, Imrich T (2008) Implementation and operational use of ground-based augmentation systems - A component of future air traffic management system. Proceedings of IEEE 96(12): 1936-1957

Pervan B, Sayim I (2001) Sigma inflation for the local area augmentation of GPS. IEEE Transactions Aerospace Electronic Systems 37(4): 1301-1311

Pervan B, Pullen S, Christie R (1998) A multiple hypothesis approach to satellite navigation integrity. The Journal of the Institute of Navigation 45(1): 61-71

RTCA DO-245, Minimum Aviation System Performance Standards for Local Area Augmentation System (LAAS). Issued 9-28-98, Prepared by SC-159

Shively C (1999) Performance analysis of alternative methods for LAAS multiple reference consistency. J Navigation 46(1):65-86

Soong T (2004) Fundamental of probability and statistics for engineer. John Wiley, New York Tang H, Pullen S, et al. (2010) Ephemeris type A fault analysis and mitigation for LAAS. In: IEEE/ION Position Location and Navigation Symposium (PLANS), CA, pp 654 - 666

Wen H, Havlicek J, Fagan J (2004) B-value research for FAA LAAS station integrity and fault detection. In :Proceedings of the 2004 National Technical Meeting of The Institute of Navigation, CA, pp 817-822

Walter T, Enge P, Blanch J, Pervan B (2008) Worldwide vertical guidance of aircraft based on modernized GPS and new integrity augmentations. Proceedings of the IEEE 96(12): 1918-1935

Wang Z, Zhang J, Zhang Q (2009) The study of integrity monitor algorithm of the pseudorange correction error on LAAS ground facility. Journal of Electronic \& Information Technology 31(1): 196-200

Author Biographies 
Liang Li is a final year PhD student at Harbin Engineering University, China. He is currently a visiting PhD student at the Department of Civil and Building Engineering at Loughborough University, UK. His research interests include: GNSS navigation and integrity monitoring.

Mohammed Quddus obtained a PhD from Imperial College London in 2005 where he was working as a research assistant for five years on a number of research projects. He joined Loughborough University as a lecturer in transport studies in 2006 and promoted to Senior Lecturer in 2010. His research interests include: sensor fusion, map-matching and the applications of statistical methods in transport.

Stephen Ison is a Professor of Transport Policy in the Department of Civil and Building Engineering, Loughborough University, UK. He is also a member of the Chartered Institute of Logistics and Transport and the Fellow of the Higher Education Academy. His research interests include: aviation, transport economics and transport demand management.

Lin Zhao is a Professor at College of Automation, Harbin Engineering University, China. He obtained his Ph.D degree in 1992 from Harbin Institute of Technology, China. His research interests include: navigation, guidance and control.

\section{Appendix A}

The mathematical induction method is used to prove $\boldsymbol{P}_{\mathrm{v}_{0, i}, k}=\boldsymbol{P}_{\mathrm{v}_{0}, k}$.

For $k=0$, we have $\boldsymbol{P}_{\mathrm{v}_{0, i}, 0}=\boldsymbol{P}_{\mathrm{v}_{0}, 0}=\overline{\boldsymbol{P}}_{0}$, which is satisfied by Eq.(13).

Assume $\boldsymbol{P}_{\mathrm{v}_{0, i}, l-1}=\boldsymbol{P}_{\mathrm{v}_{0}, l-1}$ is true for some positive integer $l$.

Then for $k=l$, we have

$$
\begin{aligned}
& \boldsymbol{\Psi}_{\mathrm{v}_{0}, l \mid l-1} \boldsymbol{P}_{\mathrm{v}_{0, i}, l-1} \boldsymbol{\Psi}_{\mathrm{v}_{i}, l \mid l-1}^{\mathrm{T}}+\boldsymbol{W}_{\mathrm{v}_{0}, l} \boldsymbol{Q}_{l} \boldsymbol{W}_{\mathrm{v}_{i}, l}^{\mathrm{T}} \\
= & \left(\boldsymbol{I}-\boldsymbol{K}_{\mathrm{v}_{0}, l} \boldsymbol{H}_{\mathrm{v}_{0}, l}\right) \boldsymbol{\Phi}_{l \mid l-1} \boldsymbol{P}_{\mathrm{v}_{0, i}, l-1} \boldsymbol{\Phi}_{l \mid l-1}^{\mathrm{T}}\left(\boldsymbol{I}-\boldsymbol{K}_{\mathrm{v}_{i}, l} \boldsymbol{H}_{\mathrm{v}_{i}, l}\right)^{\mathrm{T}}+\left(\boldsymbol{I}-\boldsymbol{K}_{\mathrm{v}_{0}, l} \boldsymbol{H}_{\mathrm{v}_{0}, l}\right) \boldsymbol{Q}_{l}\left(\boldsymbol{I}-\boldsymbol{K}_{\mathrm{v}_{i}, l} \boldsymbol{H}_{\mathrm{v}_{i}, l}\right)^{\mathrm{T}} \\
= & \left(\boldsymbol{I}-\boldsymbol{K}_{\mathrm{v}_{0}, l} \boldsymbol{H}_{\mathrm{v}_{0}, l}\right)\left(\boldsymbol{\Phi}_{l \mid l-1} \boldsymbol{P}_{\mathrm{v}_{0, i}, l-1} \boldsymbol{\Phi}_{l \mid l-1}^{\mathrm{T}}+\boldsymbol{Q}_{l}\right)\left(\boldsymbol{I}-\boldsymbol{K}_{\mathrm{v}_{i}, l} \boldsymbol{H}_{\mathrm{v}_{i}, l}\right)^{\mathrm{T}}
\end{aligned}
$$

Substitute Eq. (16) and the predetermined assumption $\boldsymbol{P}_{\mathrm{v}_{0, i}, l-1}=\boldsymbol{P}_{\mathrm{v}_{0}, l-1}$ into Eq. (A-1), we have:

$$
\boldsymbol{\Psi}_{\mathrm{v}_{0}, l \mid l-1} \boldsymbol{P}_{\mathrm{v}_{0, i}, l-1} \boldsymbol{\Psi}_{\mathrm{v}_{i}, l \mid l-1}^{\mathrm{T}}+\boldsymbol{W}_{\mathrm{v}_{0}, l} \boldsymbol{Q}_{l} \boldsymbol{W}_{\mathrm{v}_{i}, l}^{\mathrm{T}}=\boldsymbol{P}_{\mathrm{v}_{0}, l}\left(\boldsymbol{I}-\boldsymbol{K}_{\mathrm{v}_{i}, l} \boldsymbol{H}_{\mathrm{v}_{i}, l}\right)^{\mathrm{T}}
$$

According to the Kalman filter theory, the following can be obtained:

$$
\boldsymbol{K}_{\mathrm{v}_{0}, l}=\boldsymbol{P}_{\mathrm{v}_{0}, l} \boldsymbol{H}_{\mathrm{v}_{0}, l}^{\mathrm{T}} \boldsymbol{R}_{\mathrm{v}_{0}, l}^{-1}
$$

and 


$$
\begin{aligned}
\boldsymbol{K}_{\mathrm{v}_{0}, l} \boldsymbol{R}_{\mathrm{v}_{0}, l} \boldsymbol{K}_{\mathrm{v}_{i}, l}^{\mathrm{T}} & =\boldsymbol{P}_{\mathrm{v}_{0}, l} \boldsymbol{H}_{\mathrm{v}_{0}, l}^{\mathrm{T}} \boldsymbol{R}_{\mathrm{v}_{0}, l}^{-1} \boldsymbol{R}_{\mathrm{v}_{0, l}, l} \boldsymbol{K}_{\mathrm{v}_{i}, l}^{\mathrm{T}}=\boldsymbol{P}_{\mathrm{v}_{0}, l} \boldsymbol{H}_{\mathrm{v}_{0}, l}^{\mathrm{T}} \boldsymbol{R}_{\mathrm{v}_{0}, l}^{-1} \boldsymbol{R}_{\mathrm{v}_{0}, l} \boldsymbol{E}_{i} \boldsymbol{K}_{\mathrm{v}_{i}, l}^{\mathrm{T}} \\
& =\boldsymbol{P}_{\mathrm{v}_{0}, l} \boldsymbol{H}_{\mathrm{v}_{0}, l}^{\mathrm{T}} \boldsymbol{E}_{i} \boldsymbol{K}_{\mathrm{v}_{i}, l}^{\mathrm{T}}=\boldsymbol{P}_{\mathrm{v}_{0}, l} \boldsymbol{H}_{\mathrm{v}_{i}, l}^{\mathrm{T}} \boldsymbol{K}_{\mathrm{v}_{i}, l}^{\mathrm{T}}
\end{aligned}
$$

Substitute Eq. (A-4) and Eq. (A-2) into Eq. (15), we have

$$
\boldsymbol{P}_{\mathrm{v}_{0, i}, l}=\boldsymbol{P}_{\mathrm{v}_{0}, l}\left(\boldsymbol{I}-\boldsymbol{K}_{\mathrm{v}_{\mathrm{i}}, l} \boldsymbol{H}_{\mathrm{v}_{\mathrm{i}}, l}\right)^{\mathrm{T}}+\boldsymbol{P}_{\mathrm{v}_{0}, l} \boldsymbol{H}_{\mathrm{v}_{i}, l}^{\mathrm{T}} \boldsymbol{K}_{\mathrm{v}_{\mathrm{i}}, l}^{\mathrm{T}}=\boldsymbol{P}_{\mathrm{v}_{0}, l}
$$

Hence $\boldsymbol{P}_{\mathrm{v}_{0, i}, l}=\boldsymbol{P}_{\mathrm{v}_{0}, l}$ is satisfied for $k=l$.

\section{Appendix B}

Begin with Eq. (24),

$$
\begin{aligned}
& \frac{\mathrm{d} \mathrm{v}_{\mathrm{v}}}{\mathrm{d} \alpha_{\mathrm{v}_{i}}}=\frac{\mathrm{d}}{\mathrm{d} \alpha_{\mathrm{v}_{i}}}\left\{\sum_{i=0}^{3} \mathrm{P}\left(\mathrm{v}_{i}\right)\left[\int_{\hat{\boldsymbol{x}}_{\mathrm{v}}+\mathrm{VAL}}^{\infty} \mathrm{N}_{\boldsymbol{x}}\left(\hat{\boldsymbol{x}}_{\mathrm{v}_{i}}, \frac{1}{\alpha_{\mathrm{v}_{i}}^{2}} \gamma_{i}^{2} \boldsymbol{P}_{\mathrm{v}_{i}}\right) \mathrm{d} \tilde{\boldsymbol{x}}_{\mathrm{v}}+\int_{-\infty}^{\hat{x}_{\mathrm{v}}-\mathrm{VAL}} \mathrm{N}_{x}\left(\hat{\boldsymbol{x}}_{\mathrm{v}_{i}}, \frac{1}{\alpha_{\mathrm{v}_{i}}^{2}} \gamma_{i}^{2} \boldsymbol{P}_{\mathrm{v}_{i}}\right) \mathrm{d} \tilde{\boldsymbol{x}}_{\mathrm{v}}\right]\right\} \\
& \underline{\underline{s=\tilde{\boldsymbol{x}}_{\mathrm{v}}-\hat{\boldsymbol{x}}_{\mathrm{v}}}} \frac{\mathrm{d}}{\mathrm{d} \alpha_{\mathrm{v}_{i}}}\left\{\sum_{i=0}^{3} \mathrm{P}\left(\mathrm{v}_{i}\right)\left[\int_{\mathrm{VAL}}^{\infty} \mathrm{N}_{s}\left(\hat{\boldsymbol{x}}_{\mathrm{v}_{i}}-\hat{\boldsymbol{x}}_{\mathrm{v}}, \frac{1}{\alpha_{\mathrm{v}_{i}}^{2}} \gamma_{i}^{2} \boldsymbol{P}_{\mathrm{v}_{i}}\right) \mathrm{d} s+\int_{-\infty}^{-\mathrm{VAL}} \mathrm{N}_{s}\left(\hat{\boldsymbol{x}}_{\mathrm{v}_{i}}-\hat{\boldsymbol{x}}_{\mathrm{v}}, \frac{1}{\alpha_{\mathrm{v}_{i}}^{2}} \gamma_{i}^{2} \boldsymbol{P}_{\mathrm{v}_{i}}\right) \mathrm{d} s\right]\right\} \\
& \underline{\underline{u}=s \cdot \alpha_{\mathrm{v}_{i}}} \sum_{i=0}^{3} \mathrm{P}\left(\mathrm{v}_{i}\right) \frac{\mathrm{d}}{\mathrm{d} \alpha_{\mathrm{v}_{i}}}\left\{\frac{1}{\alpha_{\mathrm{v}_{i}}}\left[\int_{\alpha_{v_{i}} \cdot \mathrm{VAL}}^{\infty} \mathrm{N}_{u}\left(-\alpha_{\mathrm{v}_{i}} \beta_{\mathrm{v}_{i}}, \gamma_{i}^{2} \boldsymbol{P}_{\mathrm{v}_{i}}\right) \mathrm{d} u+\int_{\alpha_{v_{i}} \cdot \mathrm{VAL}^{\infty}}^{\infty} \mathrm{N}_{u}\left(\alpha_{\mathrm{v}_{i}} \beta_{\mathrm{v}_{i}}, \gamma_{i}^{2} \boldsymbol{P}_{\mathrm{v}_{i}}\right) \mathrm{d} u\right]\right\} \\
& =\sum_{i=0}^{3} \mathrm{P}\left(\mathrm{v}_{i}\right)\left\{\frac{1}{\alpha_{\mathrm{v}_{i}}}\left[0-\left.\mathrm{VAL} \cdot \mathrm{N}_{u}\left(-\alpha_{\mathrm{v}_{i}} \beta_{\mathrm{v}_{i}}, \gamma_{i}^{2} \boldsymbol{P}_{\mathrm{v}_{i}}\right)\right|_{u=\alpha_{v_{i}} \cdot \mathrm{VAL}}+0-\left.\mathrm{VAL} \cdot \mathrm{N}_{u}\left(\alpha_{\mathrm{v}_{i}} \beta_{\mathrm{v}_{i}}, \gamma_{i}^{2} \boldsymbol{P}_{\mathrm{v}_{i}}\right)\right|_{u=\alpha_{v_{i}} \cdot \mathrm{VAL}}\right]-\right. \\
& \left.\frac{1}{\alpha_{\mathrm{v}_{i}}^{2}}\left[\int_{\alpha_{v_{i}} \cdot \mathrm{VAL}^{\infty}}^{\infty} \mathrm{N}_{u}\left(-\alpha_{\mathrm{v}_{i}} \beta_{\mathrm{v}_{i}}, \gamma_{i}^{2} \boldsymbol{P}_{\mathrm{v}_{i}}\right) \mathrm{d} u+\int_{\alpha_{v_{i}} \cdot \mathrm{VAL}^{\infty}}^{\infty} \mathrm{N}_{u}\left(\alpha_{\mathrm{v}_{i}} \beta_{\mathrm{v}_{i}}, \gamma_{i}^{2} \boldsymbol{P}_{\mathrm{v}_{i}}\right) \mathrm{d} u\right]\right\} \\
& =\sum_{i=0}^{3} \mathrm{P}\left(\mathrm{v}_{i}\right)\left\{\frac{\mathrm{VAL}}{\alpha_{\mathrm{v}_{i}}} \cdot\left[\left.\mathrm{N}_{u}\left(-\alpha_{\mathrm{v}_{i}} \beta_{\mathrm{v}_{i}}, \gamma_{i}^{2} \boldsymbol{P}_{\mathrm{v}_{i}}\right)\right|_{u=\alpha_{v_{i}} \cdot \mathrm{VAL}}+\left.\mathrm{N}_{u}\left(\alpha_{\mathrm{v}_{i}} \beta_{\mathrm{v}_{i}}, \gamma_{i}^{2} \boldsymbol{P}_{\mathrm{v}_{i}}\right)\right|_{u=\alpha_{v_{i}} \cdot \mathrm{VAL}}\right]+\right. \\
& \left.\frac{1}{2 \alpha_{\mathrm{v}_{i}}^{2}}\left[\operatorname{erfc}\left(\frac{\alpha_{\mathrm{v}_{i}} \mathrm{VAL}+\alpha_{\mathrm{v}_{i}} \beta_{\mathrm{v}_{i}}}{\gamma_{i} \sqrt{2 \boldsymbol{P}_{\mathrm{v}_{i}}}}\right)+\operatorname{erfc}\left(\frac{\alpha_{\mathrm{v}_{i}} \mathrm{VAL}-\alpha_{\mathrm{v}_{i}} \beta_{\mathrm{v}_{i}}}{\gamma_{i} \sqrt{2 \boldsymbol{P}_{\mathrm{v}_{i}}}}\right)\right]\right\}
\end{aligned}
$$

which is equal to Eq. (25). In which $\operatorname{erfc}(x)=\frac{1}{\sqrt{\pi}} \int_{x}^{\infty} \exp \left(-t^{2}\right) \mathrm{d} t$ can be easily implemented in Matlab. 
Table 1 Explanation of four different scenarios

\begin{tabular}{ccccc}
\hline & A & B & C & D \\
\hline MLM & success & success & failure & failure \\
\hline AKFM & success & failure & success & failure \\
\hline
\end{tabular}

Table 2 Comparison of correct fault detection ratio

\begin{tabular}{ccccc}
\hline & No fault & Step fault & Slow ramp fault & Fast ramp fault \\
\hline MLM & 0.9705 & 0.6552 & 0.2524 & 0.4964 \\
\hline AKFM & 0.9818 & 0.7914 & 0.5641 & 0.7238 \\
\hline
\end{tabular}

Table 3 Positioning error after fault exclusion

\begin{tabular}{ccccc}
\hline \multicolumn{5}{c}{ CEP 95\% vertical positioning error(m) } \\
\hline & No fault & Step fault & Slow ramp fault & Fast ramp fault \\
MLM & 0.7760 & 0.9445 & 2.5440 & 1.1543 \\
AKFM & 0.1794 & 0.6443 & 0.2188 & 0.5579 \\
\hline
\end{tabular}

Table 4 Availability of each algorithm

\begin{tabular}{lcccc}
\hline \multicolumn{5}{c}{ Availability (\%) } \\
\hline \multirow{3}{*}{ MLM } & No fault & Step fault & Slow ramp fault & Fast ramp fault \\
AKFM & 92.55 & 90.88 & 52.40 & 73.92 \\
& 99.80 & 99.10 & 97.54 & 99.41 \\
\hline
\end{tabular}




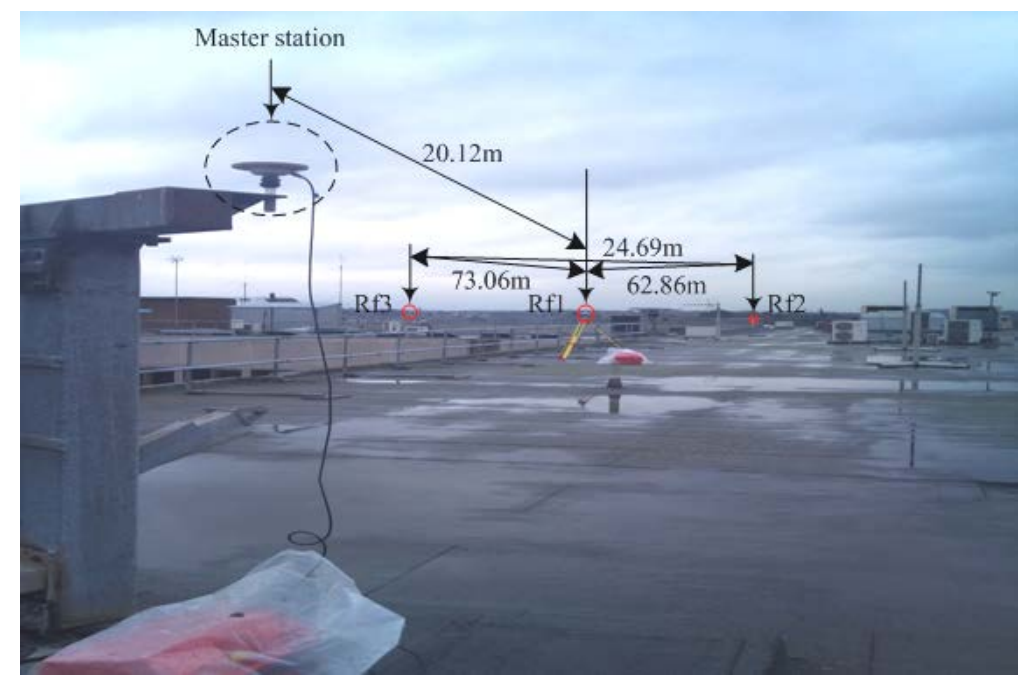

Fig. 1 The configuration of the MRCC test bed

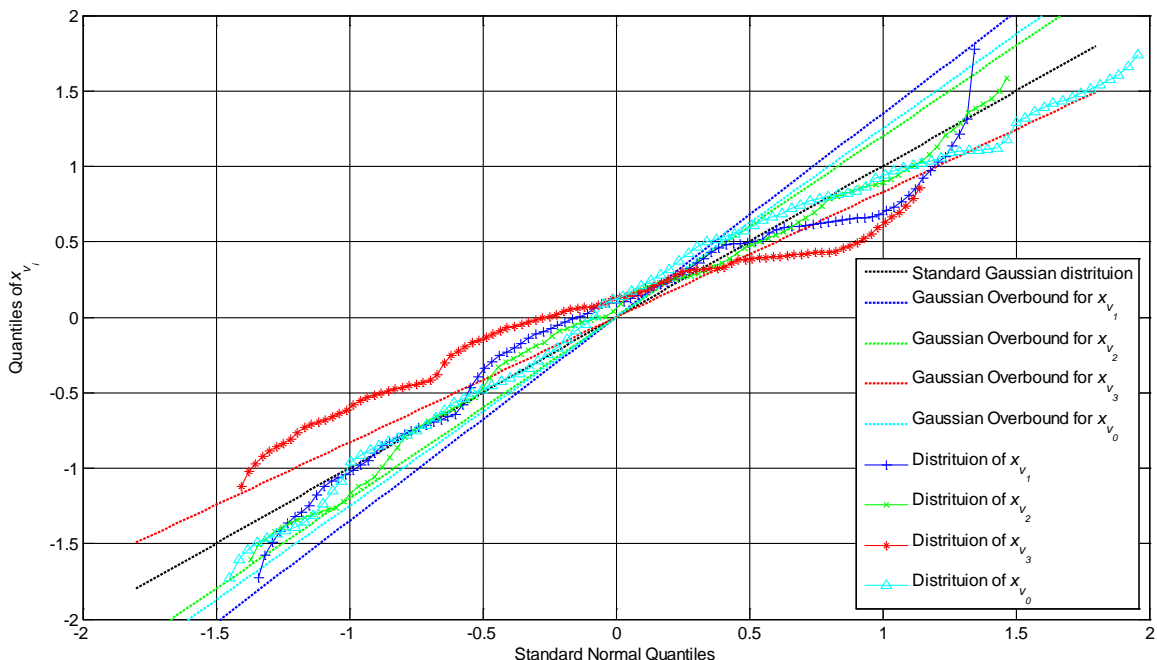

Fig. 2 The sigma inflation for the modeling errors 


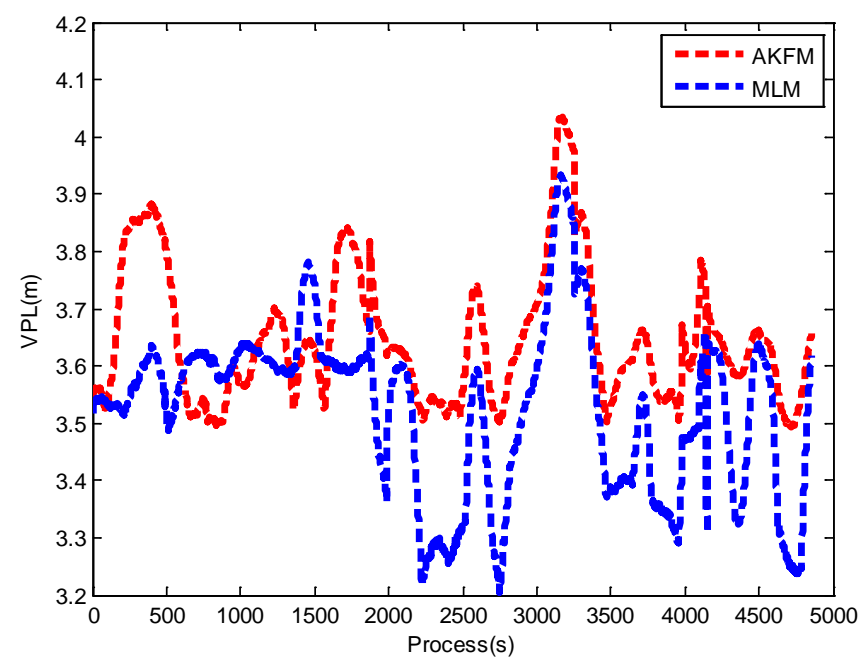

(a)

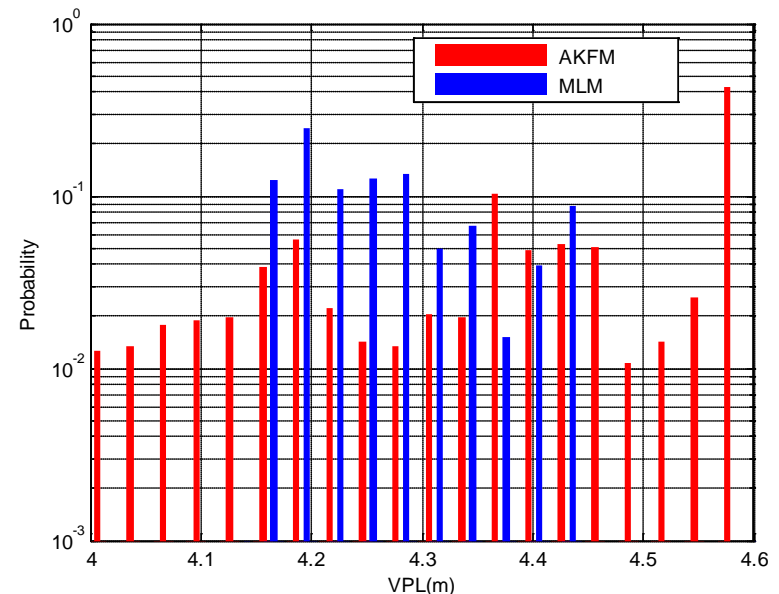

(b)

Fig. 3 a. VPL comparison between MLM and AKFM; b. VPL distribution from MLM and AKFM

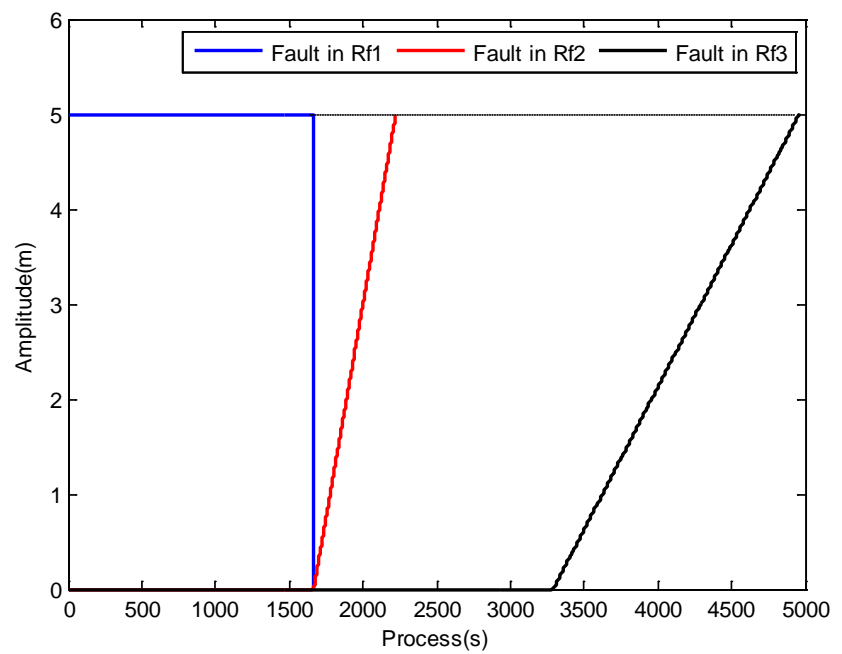

Fig. 4 Input fault scenario during the MRCC process 

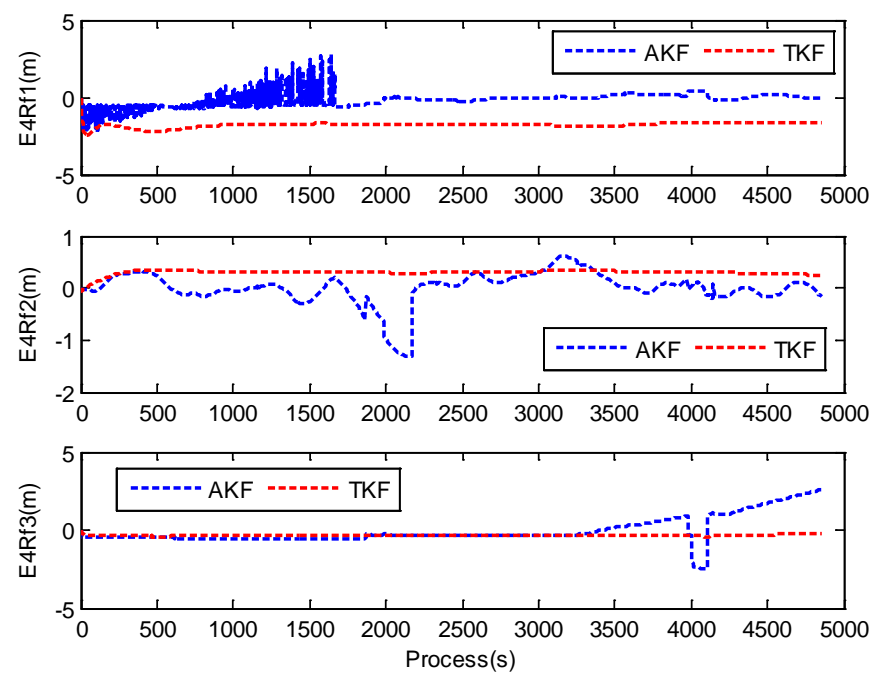

Fig. 5 The vertical positioning error from AKF and TKF
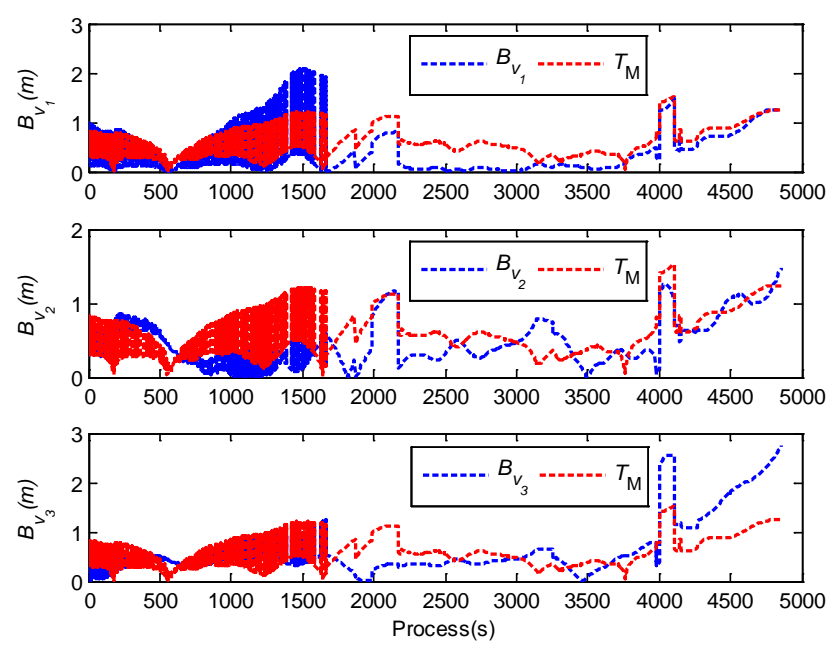

(a)
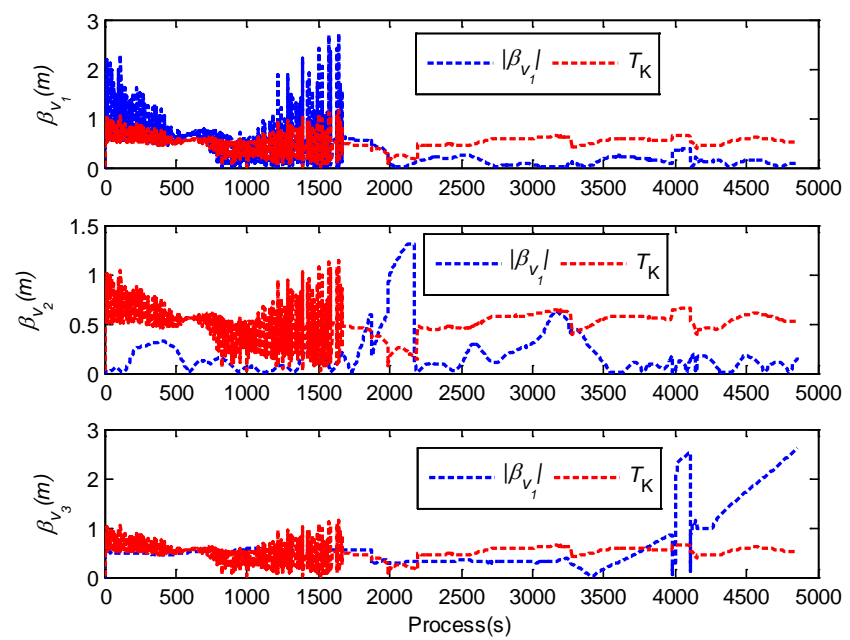

(b)

Fig. 6 a. MLM fault detection process; b. AKFM fault detection process 


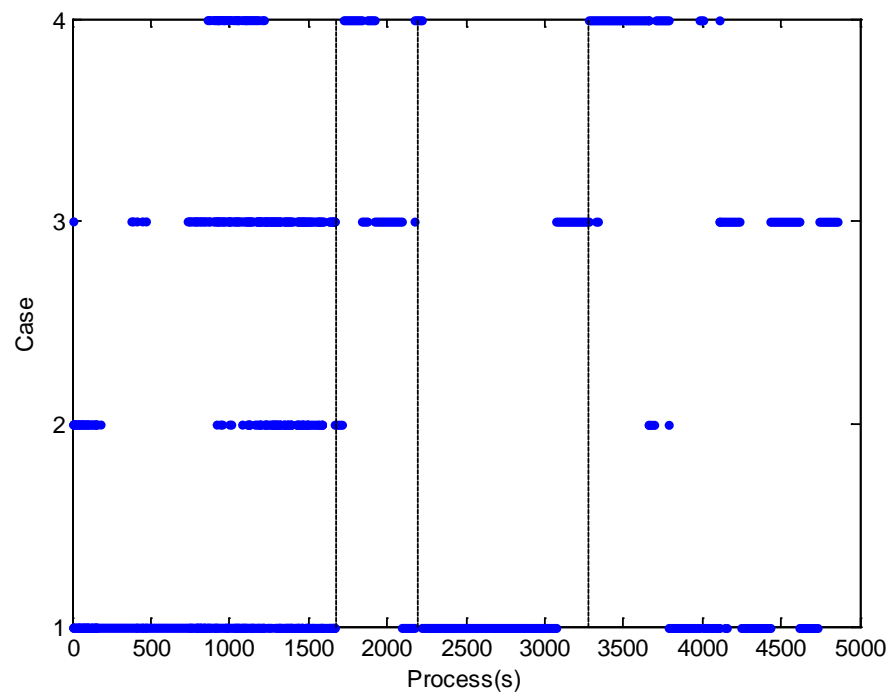

Fig. 7 Comparison of fault detection performance

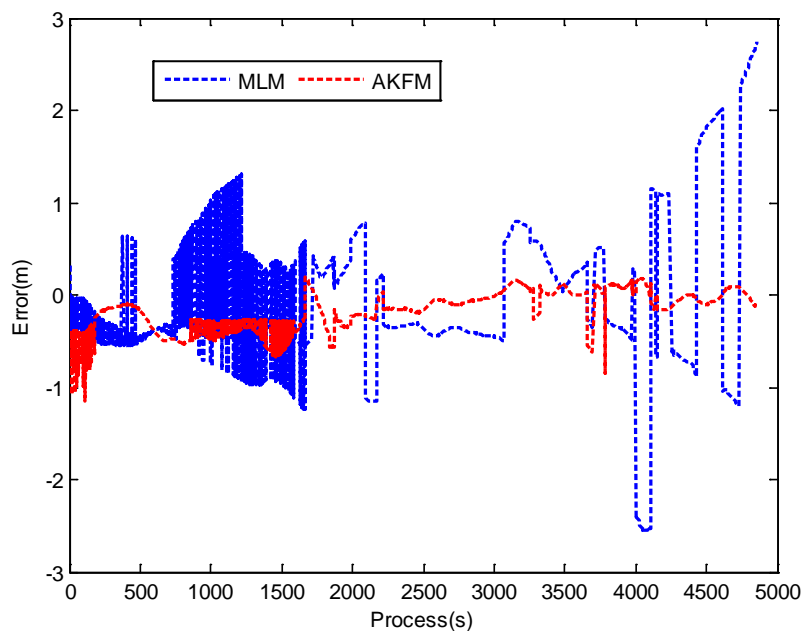

(a)

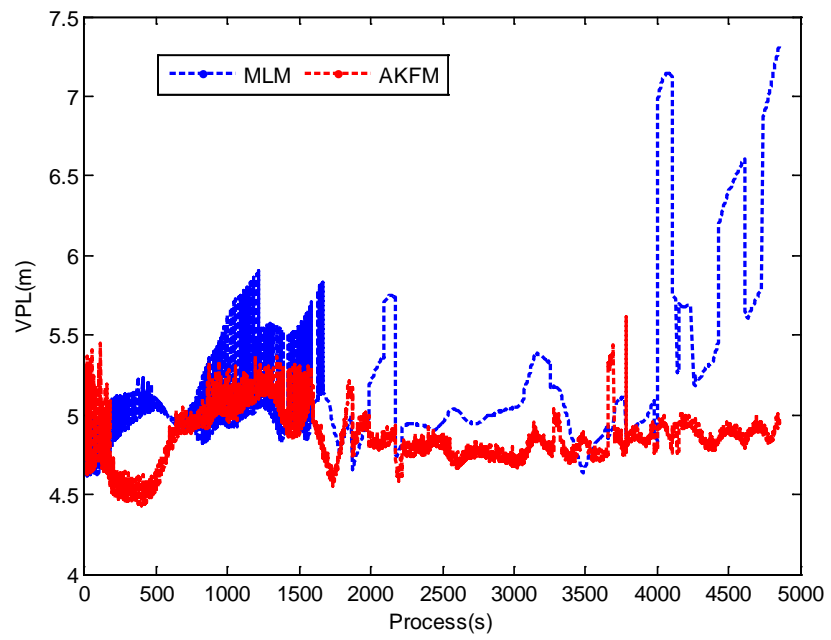

(b)

Fig. 8 a. Vertical position error after fault exclusion; b. VPL scenario after fault exclusion 